\title{
Scleromyxoedema with features of systemic sclerosis
}

\author{
Udit N Verma, R R Singh, Ramnath Misra, S Naik, S S Agarwal
}

\begin{abstract}
The case is described of a patient with scleromyxoedema with features typical of systemic sclerosis. The features were so characteristic that the disease was misdiagnosed as systemic sclerosis. A brief review of the association of the two diseases is given.
\end{abstract}

(Ann Rheum Dis 1992; 51: 1159-1161)

Since the first report of scleromyxoedema in $1954,{ }^{1}$ about 100 cases have been described. It is a sclerotic variant of papular mucinosis and is characterised by cutaneous eruptions of whitish waxy papules and plaques with variable sclerosis of the skin. Monoclonal gammopathy is the most common systemic feature associated with this disease. ${ }^{2}$ We describe here a patient with scleromyxoedema with marked overlapping features of systemic sclerosis, which led to a misdiagnosis of systemic sclerosis.

\section{Case report}

A 40 year old man presented to the immunology outpatient department with papular skin eruptions over his hands, face, neck, and chest associated with tightening of the skin, dyspnoea on exertion, and difficulty in opening the mouth and swallowing food. Twelve years before presentation he noticed small, whitish, nonpruritic cutaneous eruptions over the extensor aspects of his hands and forearms. Over the next six years these lesions gradually grew in size and spread to his arms, neck, and face. He also developed arthritis affecting his wrists, elbows, shoulders, and knees. On clinical investigation rheumatoid factor and antinuclear antibodies were absent but antibodies to pareital cells were present. He was treated with nonsteroidal anti-inflammatory drugs (NSAIDs) was subsequently added as a disease modifying over the next year, but the skin lesions persisted opening the mouth. On specific questioning he exposure to the cold, but this did not progress to cyanosis. Simultaneously he developed oesophageal hypomotility, and pulmonary function testing showed a decreased transfer factor, consistent with the diagnosis of systemic sclerosis. He was treated with D-penicillamine $(500 \mathrm{mg} /$ day $)$ and prednisolone $(60 \mathrm{mg} /$ day $)$ with partial improvement of the skin lesions, particularly tightening of the skin. Subsequently, however, the lesions progressed further and the patient stopped receiving D-penicillamine. $\mathrm{He}$ did not report any significant illness in the past and there was no similar illness in family members.

On physical examination he was $157 \mathrm{~cm}$ tall and weighed $50 \mathrm{~kg}$. His blood pressure (104/80 $\mathrm{mmHg}$ ), pulse, and respiratory rates were within the normal range. The most striking abnormality was the presence of papular skin lesions which covered extensive areas of his face, ears, the back and side of his neck, and his arms in a symmetrical manner (fig 1). Similar papules, but of a smaller size, were also present over his chest and legs. The individual papules or plaques varied from 5 to $20 \mathrm{~mm}$ in diameter, were firm in consistency, and waxy whitish in appearance. The skin was tight and hidebound, particularly over the distal extremities. Cardiovascular and respiratory examinations were normal. His abdomen was soft and there was no organomegaly. No proximal muscle weakness was noted.

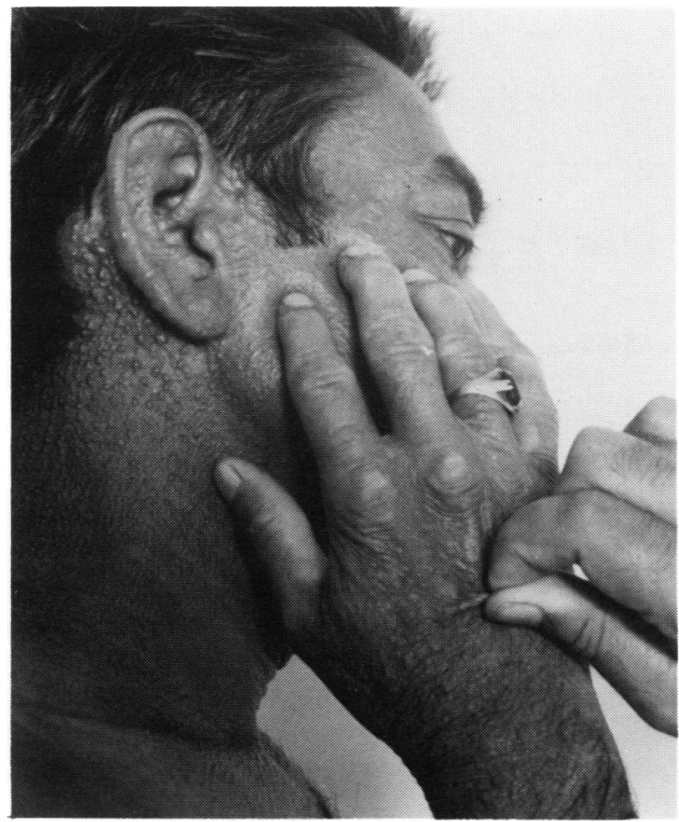

Figure 1 Dome shaped waxy papules over the neck and behind the ear, characteristic of scleromyxoedema, and sclerodactyly with thickened skin over the dorsum of the hand, which is typical of systemic sclerosis. without a satisfactory response. Chloroquine drug. The arthritic manifestations subsided with hardening of the skin and difficulty in reported that his fingertips used to turn pale on breathlessness on exertion, difficulty in swallowing food, and marked proximal muscular weakness causing difficulty in getting up from a squatting posture and in walking. Two years before presentation, he consulted another teaching institution, where a barium test showed

\author{
Post Box 375,
Lucknow 226001, India. \\ Accepted for publication \\ 25 October 1991 \\ Department of \\ Genetics, Sanjay Gand \\ of Medical Sciences, \\ S Naik \\ Correspondence to: \\ Department of Immunology \\ and Genetics, \\ Sanjay Gandhi Post-Graduate
}



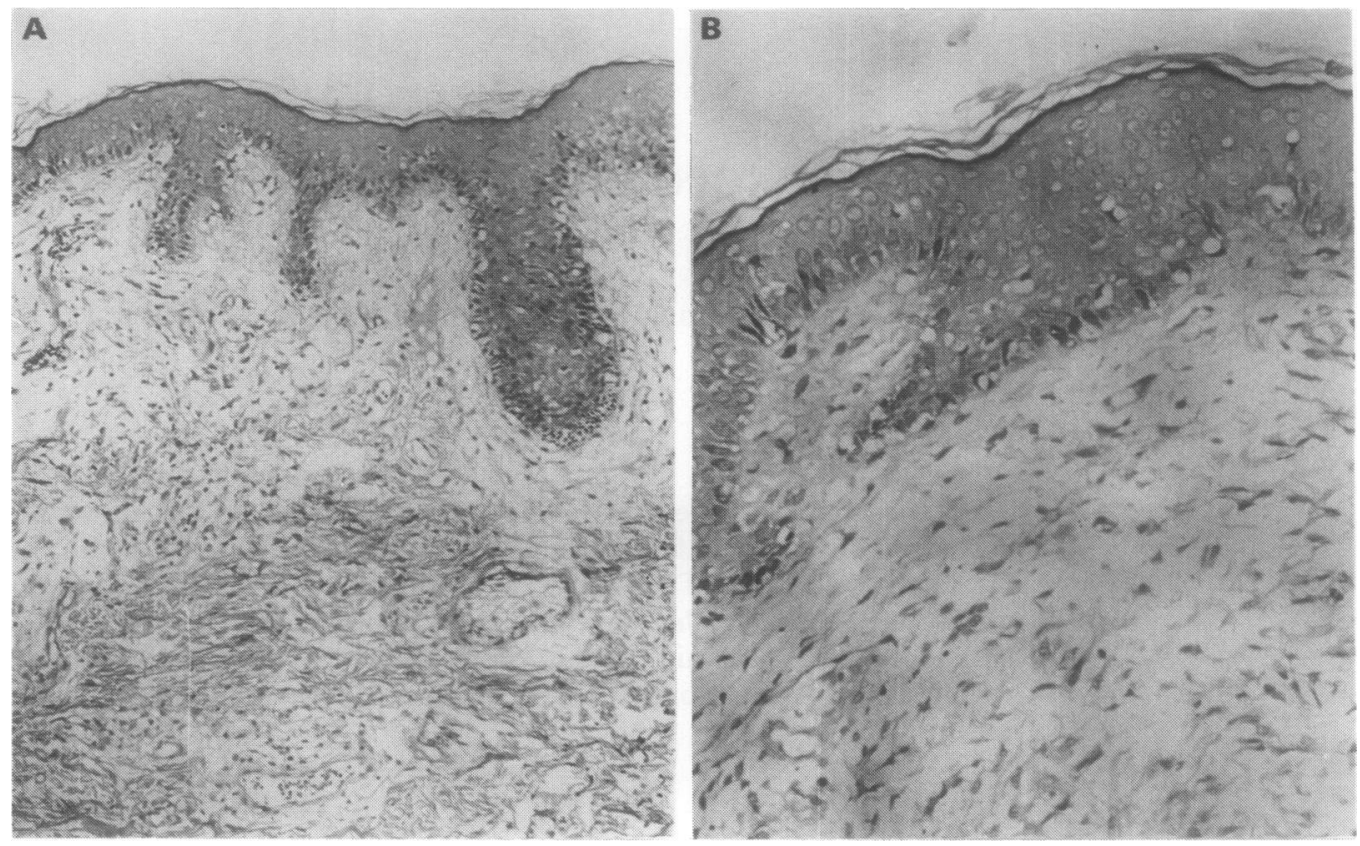

Figure 2 Histology of the skin showing a normal epidermis with an extensive focal intercellular deposit of mucin. Haematoxylin-eosin stain.

Investigations showed haemoglobin $123 \mathrm{~g} / \mathrm{l}$ and an erythrocyte sedimentation rate of $11 \mathrm{~mm}$ in the first hour (Westergren). Total and differential leucocyte counts were within normal limits. Renal function and urinary sediment were normal. Antinuclear antibodies and rheumatoid factor were negative. Antibodies to parietal cells were positive. Levels of serum complement C3 and C4 were normal. Serum IgG was increased to $36.7 \mathrm{~g} / \mathrm{l}$ with the $M$ band on serum electrophoresis. Immunofixation confirmed abnormal paraprotein to be of the gamma-lambda type. Light chains were not detected in urine samples. A bone marrow examination showed $10 \%$ plasma cells with features of hyperactivity (cytoplasmic projections and vacuolations with occasional binucleate plasma cells). A skeletal examination did not show any osteolytic areas. Oesophageal hypomotility and a respiratory function abnormality were confirmed. A chest radiograph did not show any interstitial shadows. A histological study of a skin biopsy sample showed that the epidermis was normal but there were circumscribed deposits of acid mucopolysaccharides with fibroblastic proliferation in the dermis, indicative of scleromyxoedema (fig 2).

\section{Discussion}

The case is reported of a patient with scleromyxoedema with the classical systemic manifestations of systemic sclerosis, including difficulty in opening his mouth, dysphagia, exertional breathlessness, proximal muscle weakness, polyarthritis, and Raynaud's phenomenon. The patient had hypomotility of the distal third of the oesophagus and a decreased transfer factor, typically seen in systemic sclerosis. These features were so prominent that the diagnosis of systemic sclerosis was given at a clinical immunology centre, and the patient was treated with D-penicillamine and steroids. The presence of clinically and histologically characteristic cutaneous papules and monoclonal gammopathy, however, leave no doubt that this patient had scleromyxoedema. Whether or not this patient simultaneously had the two diseases remains to be established. Tests for antibodies to $\mathrm{Scl} 70$ were not carried out, but the chances of such tests being positive are remote as tests for antinuclear antibodies were negative. A renal biopsy sample was not considered ethical as the urine sediment was normal and there was no hypertension or azotaemia. Overlaps in symptoms between scleromyxoedema and primary systemic sclerosis have been suggested previously. There have been individual case reports of patients with oesophageal hypomotility, ${ }^{3}$ scleroderma renal changes, ${ }^{45}$ and Raynaud's phenomenon with telangiectasia. ${ }^{6}$ In a few instances polymyositis ${ }^{7}$ and dermatomyositis $^{89}$ have also been reported. Although acrosclerosis is an almost constant feature of scleromyxoedema, this overlap has not resulted in any misdiagnosis. In one large series of 19 patients with scleromyxoedema from the Mayo clinic, Gabriel et al ${ }^{9}$ reported the presence of Raynaud's phenomenon in one, dysphagia in eight, dyspnoea on exertion in six, and proximal muscle weakness in five patients. Most of their patients, however, had proximal oesophageal dismotility, unlike the patient reported here, in whom the lower third of the oesophagus was affected, which is characteristic of systemic sclerosis.

There could be an aetiopathogenetic relation between the two diseases. The presence of monoclonal gammopathy and association with other B cell disorders in scleromyxoedema suggests it to be a non-neoplastic clonal disorder of the $B$ cell lineage, whereas in systemic sclerosis there is fibroblast proliferation and enhanced production of collagen. ${ }^{10}$ Whether 
paraproteins present in patients with scleromyxoedema activate the skin fibroblasts ${ }^{11}$ or B cells secrete fibrogenic cytokines which lead to the development of systemic sclerosis or similar manifestations is not known.

The optimum treatment of scleromyxoedema remains undefined. A large number of drugs, including cytotoxic drugs, have been tried. An interesting observation in our patient has been the possible response to D-penicillamine, which may be investigated in future studies.

1 Gottron H A. Skeromyxodem (Eine eigenertige Erscheinungsform von Myxothesaurodermie). Arch Dermatol Res 1954, 199: 71-91.

2 McCarthy J T, Osserman E, Lombardo P C, Takatsuki K. An abnormal serum globulin in lichen myxedematosus. Arch Dermatol 1964; 89: 446-50.
3 Alligood T R, Burnett J W, Raines B L. Scleromyxoedema associated with oseophageal aperistalsis and derma eosinophilia. Cutis 1981; 28: 60-4.

4 Kantor G R, Bugfeld W F, Katzin W E, et al. Scleromyxoedema associated with scleroderma renal disease and acute psychosis. F Am Acad Dermatol 1986; 14: 879-88.

5 Harris R B, Perry H O, Kyle R A, Winkelmann R K. Treatment of scleromyxoedema with melphalan. Arch Dermatol 1979; 115: 295-9.

6 Fudman E J, Globus J, Ike R W. Scleromyxoedema with systemic involvement mimics rheumatic diseases. Arthritis Rystemic involvement mim 1986; 29: 913-7.

7 Kuba R. Champion R H. Scleromyxoedema. $B r f$ Dermatol 1975; 93 (suppl): 36-8.

8 Johnson B L, Horowitz I R, Charles C R, Cooper D L. Johnson B L, Horowitz I R, Charles C R, Cooper D L.
Dermatomyositis and lichen myxoedematosus. Dermatologica 1973; 147: 109-22.

9 Gabriel S E, Perry H O, Oleson G B, Bowles G A Scleromyxoedema: a scleroderma like disorder with systemic manifestations. Medicine (Baltimore) 1988; 67: 58-65.

10 Raghu G, Chen Y, Rusch V, et al. Differential proliferation of fibroblast cultured from normal and fibrotic lungs. Am Rev Respir Dis 1988; 138: 703-8.

11 Harper R A, Rispler J. Lichen myxoedematosus serum stimulates human skin fibroblast proliferation. Science 1978; 199: 545-7. 